\title{
Disease trajectories in behavioural variant frontotemporal \\ dementia, primary psychiatric and other neurodegenerative disorders presenting with behavioural change
}

\author{
Lianne M Reus ${ }^{1 *}$, Everard GB Vijverberg ${ }^{1}$, Betty M Tijms ${ }^{1}$, Mara ten Kate ${ }^{1}$, Flora Gossink ${ }^{2}$, \\ Welmoed A Krudop ${ }^{1,3}$, Marta del Campo ${ }^{4}$, Charlotte E Teunissen ${ }^{4}$, Frederik Barkhof ${ }^{5,6}$, \\ Wiesje M van der Flier ${ }^{1}$, Pieter Jelle Visser ${ }^{1,7}$, Annemiek Dols ${ }^{2}$, Yolande AL Pijnenburg ${ }^{1}$ \\ ${ }^{1}$ Department of Neurology and Alzheimer centre, VU University Medical Centre, Amsterdam \\ Neuroscience, Amsterdam, The Netherlands. \\ ${ }^{2}$ Department of Old Age Psychiatry, GGZ inGeest, Amsterdam, The Netherlands. \\ ${ }^{3}$ Department of Psychiatry, UMC Utrecht, Utrecht, The Netherlands.
}

${ }^{4}$ Neurochemistry Lab and Biobank, Department of Clinical Chemistry, VU University Medical Centre, Amsterdam Neuroscience, Amsterdam, The Netherlands.

${ }^{5}$ Department of Radiology and Nuclear Medicine, VU University Medical Centre, Amsterdam, The Netherlands.

${ }^{6}$ Institutes of Neurology and Healthcare Engineering, UCL, London, United Kingdom.

${ }^{7}$ Department of Psychiatry, Maastricht University, Maastricht, The Netherlands.

* Correspondence to Lianne Maria Reus, De Boelelaan 11171081 HV Amsterdam, I.reus@vumc.nl, $+31(0) 204440685$

Word count: 246/3465 (abstract/main text) Figures/tables: 1/4 Supplementary figures/tables: 0/7 


\section{ABSTRACT}

Behavioural variant frontotemporal dementia (bvFTD) is characterized by behavioural and social cognitive disturbances, while various psychiatric and neurodegenerative disorders may have similar clinical symptoms. Since neurodegenerative disorders are eventually progressive, whereas primary psychiatric disorders are not, this study aimed to investigate whether the change in clinical symptoms over time differed between groups and which biomarkers predicted rate of decline.

Disease trajectories (median follow-up=3 years) of frontal and stereotyped behaviour, general and frontal cognitive functioning, and social cognition were examined in bvFTD $(n=34)$, other neurodegenerative $(n=28)$ and primary psychiatric disorders $(n=43)$, all presenting with late-onset frontal lobe syndrome (45-75 years), using linear mixed models. To gain more insight in underlying pathological processes driving disease progression, we studied the association of baseline cerebrospinal fluid (CSF) (neurofilament light ( $\mathrm{NLL}$ ) and YKL-40 levels, phosphotau ${ }_{181}$ to total tau ratio) and neuroimaging markers with disease trajectories.

Frontal behavioural symptoms (e.g., disinhibition, apathy) worsened over time in bvFTD, whereas they improved in psychiatric disorders and remained stable in other neurodegenerative disorders. General and frontal cognitive decline was observed in bvFTD and other neurodegenerative disorders, but not in psychiatric disorders. None of the groups showed change in stereotypy and social cognition. For all diagnostic groups, higher CSF NfL levels were associated with faster frontal cognitive decline. A modest association was observed between caudate volume and stereotyped behaviour. 
Tracking frontal behavioural symptoms and cognition has potential to distinguish bvFTD from other disorders. CSF NfL levels seem to be associated with decline in frontal cognitive functioning.

Key words: behavioural variant frontotemporal dementia (bvFTD), disease trajectories, cerebrospinal fluid, magnetic resonance imaging (MRI), subcortical volumes, cortical thickness 


\section{INTRODUCTION}

Behavioural variant frontotemporal dementia (bvFTD) is a neurodegenerative disorder presenting with behavioural changes and deterioration of social cognition(Rascovsky et al., 2011). However, these symptoms can also be observed in various psychiatric and other neurodegenerative disorders, such as major depressive disorder and Alzheimer's disease (AD),(Pose et al., 2013; Woolley et al., 2011). It is important to differentiate between these disorders as clinical management will be different.

We have set up the late-onset frontal lobe syndrome (LOF) study, including individuals who presented with behavioural changes during middle to late adulthood at a memory clinic setting(Krudop et al., 2014). In earlier studies using baseline data from this cohort we have shown that impaired emotion recognition and the presence of stereotypy are suggestive of a diagnosis of bvFTD, while theory of mind and cognitive performances did not discriminate bvFTD from other disorders presenting with late-onset behavioural change,(Dols et al., 2016; Gossink et al., 2017; Krudop et al., 2015; Vijverberg, et al., 2017).

The present study aimed to investigate whether the change in behavioural, cognitive and social cognitive symptoms over time differed between diagnostic groups, and which biomarkers predicted rate of decline. Most prospective studies in bvFTD examined cognitive decline,(Boutoleau-Bretonniere et al., 2012; O'Connor et al., 2016; Smits et al., 2015; Tan et al., 2013), but not the progression of behavioural and social cognitive symptoms(Kumfor et al., 2014). The only study so far focussing on these measures has shown that bvFTD patients decline more rapidly on emotion recognition compared to AD patients(Kumfor et al., 2014). We hypothesize that disease progression is faster in bvFTD compared to primary psychiatric disorders, as neurodegenerative disorders are progressive and eventually terminal, whereas 
psychiatric disorders overall are not. Although other neurodegenerative disorders than bvFTD lack the specific frontotemporal distribution of pathology, in some cases clinical dysfunction related to frontotemporal areas may be predominant. The rate of change of clinical measures will mostly depend on the rate of neurodegeneration. To date too little information is available about the rate of decline in these atypical variants. It would therefore be difficult to hypothesize about the potential differences between bvFTD and other neurodegenerative diseases.

In order to better understand the underlying pathology driving disease progression, we tested which biomarkers could predict rate of decline. We selected subcortical volumes, frontal and temporal brain atrophy, as estimated by cortical thickness measurements, and decreased cerebrospinal (CSF) phosphorylated tau $\mathrm{u}_{181}$ to total tau ( $\mathrm{p} / \mathrm{t}$-tau) ratio and increased CSF neurofilament light (NfL) and YKL-40 protein levels, biomarkers which all have been associated with bvFTD in previous studies,(Dolan, 1999; Meeter et al., 2016; Pijnenburg et al., 2015; Teunissen et al., 2016; Vijverberg, et al., 2017). CSF NfL and p/t-tau ratio reflect axonal degeneration and CSF YKL-40 is an inflammatory marker,(Meeter et al., 2016; Pijnenburg et al., 2015; Teunissen et al., 2016; Vijverberg, et al., 2017). Higher levels of brain atrophy in bVFTD have been associated with faster decline on emotion recognition and cognitive function,(Borroni et al., 2012; Josephs et al., 2011; Kumfor et al., 2014; Ranasinghe et al., 2016). Decreased CSF p/t-tau ratio and increased NfL levels have been associated with poorer prognosis across FTD subtypes(Pijnenburg et al., 2015). Therefore, we hypothesize that more abnormal biological values would predict faster decline. 


\section{METHODS}

\section{Patients}

137 patients $\left(n_{\text {male }} / n_{\text {female }}=98 / 39\right.$, mean \pm SD age $\left.=62 \pm 7\right)$ participated in the late-onset frontal lobe syndrome (LOF) study, which is a multi-centre observational and prospective follow-up study designed to examine the discrimination of bvFTD from other disorders presenting with similar clinical presentations, such as apathy, disinhibition, and/or compulsive stereotypical behaviour(Krudop et al., 2014). The LOF study is a naturalistic follow-up cohort study, thereby not preventing treatment (e.g., medication or cognitive therapy in the psychiatric group). Patients with a late onset (45 - 75 years) frontal lobe syndrome, defined as a clinical syndrome associated with functional or structural changes in the prefrontal cortex, leading to personality, affective or behavioural changes(Krudop and Pijnenburg, 2015), were recruited from the memory clinic of the Alzheimer Center VUmc and the Old Age Psychiatry Department of GGZ inGeest, Amsterdam, The Netherlands, between April 2011 and June 2013. Inclusion and exclusion criteria of the LOF study have been described elsewhere in detail(Krudop et al., 2014). In short, patients were included when behavioral symptoms dominated the clinical presentation, the score on the Frontal Behavioural Inventory (FBI) was $\geq 11$ or the Stereotypy Rating Inventory (SRI) score was $\geq 10$, and the Mini-Mental State Examination (MMSE)-score was $\geq 18$.

At baseline and after two years, a consensus diagnosis was made by a specialized neurologist and psychiatrist during a multidisciplinary meeting(Vijverberg, et al., 2017). Two-year followup diagnoses were used as gold standard. As the present study aimed to compare disease trajectories across probable/definite bvFTD, primary psychiatric and other neurodegenerative disorders, patients with an unknown diagnosis after two years of follow- 
up $(n=1)$ or other diagnoses, such as subjective complaints $(n=5)$, psychological problems $(n=1)$, relational/marital problems $(n=6)$, possible bvFTD $(n=5)$, other neurological or general diseases (e.g., multiple sclerosis, $n=10$ ) or vascular mild cognitive impairment $(n=4)$, were excluded.

The analysis was conducted in 105 patients ( $n_{\text {bvFTD }}=34, n_{\text {psychiatric diagnosis }}=43, n_{\text {neurodegenerative }}$ diagnosis=28). At 2-year follow-up, 30 patients were diagnosed as having probable bvFTD and four as definite bvFTD (i.e., two C9orf72 expansion hexanucleotide repeat, one progranulin mutation and one histopathologically-confirmed tauopathy). In the primary psychiatric disorder group, 15 patients were diagnosed with major depressive disorder, 7 with bipolar disorder, 8 with personality disorder, 6 with minor depressive disorder, 3 with autism spectrum disorder, 1 with schizoaffective disorder, 1 with schizophrenia, 1 with obsessive compulsive disorder and 1 patient with anxiety disorder. The neurodegenerative diagnosis group consisted of 8 patients with Alzheimer's disease, 4 with vascular dementia, 4 with dementia with Lewy bodies, 5 with progressive supranuclear palsy, 3 with semantic dementia, 2 with neurodegenerative syndrome not otherwise specified, 1 with Huntington's disease, and 1 patient with corticobasal syndrome. The number of measurements was different between diagnostic groups for the majority of cognitive measures (Table 1), whereas follow-up duration did not differ across diagnostic groups (Table S1).

Informed consent, either from the patient or from the legal representative, was obtained from all participants. This study was approved by the Medical Ethics Committee of the VU University Medical Centre, Amsterdam. 


\section{Diagnostic work-up}

At baseline, all patients received a standardized multidisciplinary assessment, consisting of medical history, informant-based history, neurological and medical examination, neuropsychological investigation, brain magnetic resonance imaging (MRI), standard laboratory work-up and lumbar puncture(Van der Flier, 2014). Diagnoses were based upon the psychiatric(Association;, 2000) and neurodegenerative disease guidelines,(McKeith et al., 2005; McKhann et al., 2011; Rascovsky et al., 2011; Roman et al., 1993). After 2-year followup, neurological and medical examination, neuropsychological investigation and brain MRI were repeated. After two years of follow-up, the presence of a hexanucleotide repeat expansion in C9orf72 was examined for $n=97$ participants(Galimberti et al., 2013). In case of a positive family history, patients were referred to the clinical genetics department, where screening for other genetic mutations (MAPT, Progranulin, Presenilin 1) was offered.

\section{Clinical and neuropsychiatric assessment}

Clinical assessments were assessed at baseline and at 1-year and 2-year follow-up. Severity of frontal behavioural symptoms was rated using the informant-based $\mathrm{FBI}$, which consists of negative (e.g., apathy, indifference, loss of insight) and positive (e.g., inappropriateness, hyper-orality, aggression) subscales(Andrew, 2000). The severity of stereotyped behaviour on eating and cooking behaviours, roaming, speaking, movements and daily rhythm was estimated using the informant-based SRI(Shigenobu et al., 2002).The MMSE and Frontal Assessment Battery ( $F A B)$ were assessed to estimate general cognitive and frontal lobe functioning, respectively,(Dubois et al., 2000; Folstein et al., 1975).

Neuropsychological testing was performed at baseline and 2-year follow-up. Emotion recognition and theory of mind were measured using the Ekman 60(Diehl-Schmid et al., 
2007) and Faux Pas test(Baron-Cohen et al., 1999). For the Ekman 60 test, patients have to indicate the expressed emotion of 60 displayed faces (ten actors [ $40 \%$ males] of the Facial Expression of Emotion: Stimuli and Tests (FEEST)), consisting of the basic emotions anger, disgust, fear, sadness, surprise and happiness. Prior to testing, it was verified that all patients semantically understood these six emotions. During the Faux pas test, stories with or without a Faux Pas (i.e., an embarrassing social mistake) were presented to the patient. The patient was asked to indicate for each story whether there was a Faux Pas (yes/no). To verify whether the patient used a correct rationale, the patient had to indicate the emotion of the character in the story (empathy score). Lower scores on the Faux pas reflect worse performance.

\section{CSF analysis}

CSF was obtained using a 25-gauge needle and syringe. A quantity of approximately $12 \mathrm{~mL}$ was collected in polypropylene tubes (Corning Life Sciences) and within one hour centrifuged at $1800 \mathrm{~g}$ for 10 minutes at $4^{\circ} \mathrm{C}$ (Teunissen et al., 2009). Supernatants were stored at $-20^{\circ} \mathrm{C}$ for routine biomarker analysis (t-tau, $\mathrm{p}$-tau), and aliquots from separate tubes were biobanked at $-80^{\circ} \mathrm{C}$ for other biomarker studies. CSF levels of $\mathrm{NfL}$ and $\mathrm{YKL}-40$ were determined using ELISAs according to the manufacturer's instructions (Uman Diagnostics Umea, Sweden; MicroVueTM YKL-40 EIA kit, Quidel, CA, USA). Performance of the assays was evaluated using CSF pools as internal controls. Intra- and inter- assays CVs were for NfL 1.3\% and $6.1 \%$ and for YKL40 2.8\% and 9.5\%. CSF levels of total tau (t-tau) and phosphotau181 tau (p-tau) were determined on a routine basis, using enzyme-linked immunosorbent assay (Innotest: Fujirebio, Ghent, Belgium). Interassay CVs were $9.9 \pm 2.1 \%$ for t-tau, and $9.1 \pm 1.8 \%$ for $p$-tau. 
CSF measurements of $\mathrm{NfL}$ were available for $n=60, Y K L-40$ for $n=63$ and tau forms for $n=77$ individuals. Reasons for missing CSF parameters were technical issues ( $n=16$, such as low CSF volume), patient refusal to undergo lumbar puncture $(n=9)$ or contra-indication for lumbar puncture (use of anticoagulants) $(n=3)$.

\section{MRI acquisition and pre-processing}

T1-weighted structural scans used in the current study were acquired at baseline on either a 3T General Electric (GE) Signa ( $n=45$ ) or 3T Phillips PET-MR $(n=8)$ scanner equipped with a standard head eight-channel coil at the VU University Medical Centre (see supplementary materials for more details). Imaging parameters for the GE Signa scanner were TR=7.84 msec, TE=3.02 msec, flip angle $=12^{\circ}$, slice thickness=1, 176 slices. Imaging parameters for the Philips scanner were: $T R=9.89 \mathrm{msec}, \mathrm{TE}=4.59 \mathrm{msec}$, flip angle $=8^{\circ}$, slice thickness $=1,180$ slices. Reasons for lacking MR scans for the other patients $(n=32)$ included MR contraindications or MR scans being acquired on a scanner with a field strength below 3T $(n=20)$. Patients scanned on the GE and Philips scanner, and patients with and without available MR scans, were compared in age, gender and diagnosis, to exclude the possibility that demographic and clinical variables were different between these groups (results presented in the Supplementary materials). Cortical thickness measures and subcortical volumes were extracted from T1-weighted images using FreeSurfer v5.3.0 (http://surfer.nmr.mgh.harvard.edu/, (Fischl and Dale, 2000; Fischl et al., 2004; Segonne et al., 2004; Segonne et al., 2007)). Cortical areas were summarised in 73 regions according to the Desikan-Killiany atlas(Desikan et al., 2006). Cortical thickness measures and subcortical volumes were extracted for $n=48$ subjects ( $n=40$ GE [13 bvFTD, 10 psychiatry, 17 neurodegenerative], n=8 Phillips [2 bvFTD, 2 psychiatry, 4 neurodegenerative]). Segmentations were visually inspected, including tissue identification and boundary position 
errors, which resulted in exclusion of $n=5$ (GE scanner) subjects. Cortical thickness values of five frontal and eight temporal areas were used in the current analysis. Neuroimaging measures were corrected for age, gender, total intracranial volume (for subcortical volume only) and scanner type with linear regression models. The residuals of these models are considered to be covariate-corrected neuroimaging estimates.

\section{Statistical analysis}

Analyses were performed using Statistical Package for the Social Sciences $\left(\right.$ IBM $^{\circledR}$, SPSS $^{\circledR}$ Statistics, V22, Chicago, Illinois) and R (version 3.2.5, R Development Core Team, 2010). To assess group differences at baseline, univariate analysis of variance (ANOVA), $\chi^{2}$, and Kruskal-Wallis $\mathrm{H}$ tests were used where appropriate. To examine whether patients who dropped out of the study where different than those who did not, multinomial logistic regression analyses were performed in which we examined whether the number of followup visits was associated with clinical measures. Baseline scores on the FBI, SRI, MMSE, FAB, Faux pas total and Ekman tests were not related to drop-out rate (Table S1).

Longitudinal changes over time were assessed with linear mixed models. The model included terms for time (years), diagnostic group and an interaction term of diagnosis*time. For the $\mathrm{FBI}, \mathrm{MMSE}, \mathrm{FAB}$ and Faux pas test, a fixed intercept and a random slope with time were assumed, meaning that the model accounted for individual variation of change in the scale of interest over time, whereas baseline measures were not allowed to vary between patients. For the SRI and Ekman faces test a random intercept was assumed, as baseline values for these questionnaires have previously been found differ between diagnostic groups,(Dols et al., 2016; Gossink et al., 2017; Krudop et al., 2015; Vijverberg, et al., 2017). Abovementioned analyses were performed without (model 1) and with (model 2) the 
covariate adjustment for age, gender and level of education (years). As genetic forms of FTD (e.g., C9orf72repeat expansion) can present with different phenotypes, analyses were repeated excluding these cases $(n=3)$ (Galimberti et al., 2013). Results did not change after excluding FTD patients with a genetic mutation (Table S2 and S3).

We further tested whether neuroimaging or CSF measures were predictive of changes over time in clinical measures, by adding a three-way interaction term to the models (i.e., diagnosis*time*biological predictor). If this three-way interaction was found to be significant, time*biological predictor associations were stratified across diagnostic groups. In case of no significant three-way interaction, analyses were repeated with biological predictor*time.

For all homologue brain regions, associations between progression and neuroimaging measure were examined using linear mixed models, modelling hemisphere and time as random factors. Neuroimaging results were corrected for multiple comparisons (i.e., seven subcortical volumes and thirteen cortical thickness areas) using a false discovery rate (FDR) correction. A p value $\leq 0.05$ was interpreted as significant. 


\section{RESULTS}

\section{Baseline characteristics}

Table 1 displays the demographic characteristics per diagnostic group. Diagnostic groups had similar gender distributions, levels of education and disease duration. Psychiatric patients were younger compared to patients with a neurodegenerative disorder $(p<0.05)$. BvFTD patients showed higher levels of stereotypy and lower performances on emotion recognition at baseline. Baseline higher CSF NfL and YKL-40 levels, and lower p/t-tau ratios were observed in bvFTD patients (bvFTD vs. psychiatry; $p_{\mathrm{NFL}}<0.001, \mathrm{p}_{\mathrm{YKL}-40}<0.001, \mathrm{p}_{\mathrm{p} / \mathrm{t} \text {-tau ratio }}<0.001$; bvFTD vs. neurodegenerative; $\left.p_{\mathrm{NfL}}<0.05, \mathrm{p}_{\mathrm{YKL}-40}<0.05, \mathrm{p}_{\mathrm{p} / \mathrm{t} \text {-tau ratio }}<0.05\right)$, followed by patients with a neurodegenerative disorder (neurodegenerative vs. psychiatry; $\mathrm{p}_{\mathrm{NfL}}<0.05, \mathrm{p}_{\mathrm{YKL}-40}<0.05$,

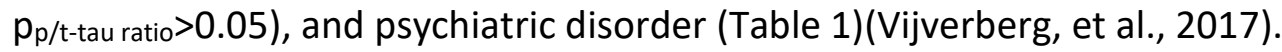

\section{Behavioural change}

Progression over time on frontal behavioural symptoms, as measured with the $\mathrm{FBI}$, was different between diagnostic groups (interaction: $\mathrm{F}=8.513, \mathrm{p}<0.001$ ), with symptoms worsening in bvFTD $(B(S E)=2.902(1.124), \quad p<0.05)$, remaining stable in other neurodegenerative disorders $(B(S E)=-0.526(1.218), p>0.05)$ and diminishing in psychiatric disorders $(B(S E)=-3.536(0.956), p<0.001)($ Figure $1 A$, Table 2, Table 3). These results seemed to be driven by the negative subscale (interaction: $F=9.557, p<0.001$ ), rather than by the positive subscale (interaction: $\mathrm{F}=2.370, \mathrm{p}>0.05$ )(Table 2 ).

Trajectories on stereotypical behaviour (SRI) did not change over time (main effect time: $\mathrm{F}=0.378, \mathrm{p}>0.05$ ) and were not different between the diagnostic groups (interaction: $F=1.627, p>0.05$ )(Figure 1B, Table 2, Table 3). 


\section{Decline in social cognitive functioning}

The longitudinal trajectories of emotion recognition (Ekman 60) did not change over time (main effect time: $\mathrm{F}=0.224, \mathrm{p}>0.05$ ) and did not differ between diagnostic groups (interaction: $\mathrm{F}=0.833, \mathrm{p}>0.05$ )(Figure $1 \mathrm{E}$, Table 2, Table 3). Exploratory analyses on facial expressions separately revealed no associations (all $p>0.05$ )(Table S4).

Faux pas correct subscores increased over time averaged across diagnostic groups (main effect time: $\mathrm{F}=5.465, \mathrm{p}<0.05)$, but did not differ between diagnostic groups (interaction: $\mathrm{F}=0.833, \mathrm{p}>0.05)$. Performances on empathy faux pas subscores did not change over time (main effect time: $\mathrm{F}=0.035, \mathrm{p}>0.05$ )(Figure $1 \mathrm{~F}$, Table 2, Table 3).

\section{Cognitive decline}

Rate of decline differed between groups for both the FAB (interaction: $F=7.020, p<0.01$ ) and MMSE (interaction: $F=4.547, p<0.05)$. BvFTD patients declined on both the $F A B(B(S E)=-1.624$ (0.448), $p<0.05)$ and MMSE $(B(S E)=-1.538(0.540), p<0.05)$, whereas patients with a neurodegenerative disorder declined on the MMSE only $(F A B: B(S E)=-0.315(0.337), p>0.05$, MMSE: $(B(S E)=-1.144(0.417), p<0.05)($ Figure $1 C$ and $D$, Table 2, Table 3). Patients with a psychiatric disorder did not decline on the MMSE $(B(S E)=0.095(0.332), p>0.05)$ nor on the $F A B(B(S E)=0.312(0.262), p>0.05)$

\section{Baseline neuroimaging and CSF measures in relation to disease progression}

Neuroimaging markers were not associated with rate of decline on any clinical measure. For all biological markers, no significant three-way interactions were observed, indicating that associations between baseline value and cognitive change are not different across diagnostic groups. Neuroimaging differences between diagnostic groups are presented in supplementary Table S5. Smaller caudate volumes at baseline were nominally associated 
with more stereotyped behaviour (SRI) over time $(B(S E)=0.004(0.002)$, puncorr $=0.059)$. Nevertheless, this association did not survive correction for multiple comparisons $\left(p_{F D R}>0.05\right)(T a b l e ~ 4)$. Cortical thickness measurements were not associated with disease progression(Table 5).

Across diagnostic groups, higher NfL CSF levels at baseline were associated with a steeper rate of decline on frontal lobe functioning $(F A B)$ over time $(B=-0.001(0.000), p<0.05)$. No other significant associations were found between baseline CSF measures and disease progression(Table 6). 


\section{DISCUSSION}

In the current study, we examined the longitudinal trajectories of bvFTD in comparison to other neurodegenerative conditions and primary psychiatric disorders presenting with lateonset behavioural change. Our results provide strong evidence for faster worsening of frontal behavioural symptoms, measured with FBI, in bvFTD compared to other neurodegenerative and psychiatric disorders. Deterioration of frontal lobe cognitive functioning and general cognition, as estimated with the FAB and MMSE, was observed in bvFTD and other neurodegenerative disorders, with a faster decline on the FAB observed in bvFTD. There were no differences between diagnostic groups in longitudinal social cognitive decline, measured using Ekman faces and Faux pas tests.

Our results suggest that positive symptoms, such as inappropriateness, hyper-orality, aggression, and stereotyped behaviour are present in the early phase of bvFTD, as positive FBI scores at the initial visit have been previously shown to be different between diagnostic groups (Dols et al., 2016; Krudop et al., 2015) - but may not change any further over the time period studied in this study, the diagnostic stage. Since our follow-up duration was 2 years, I cannot be excluded that an increase of positive symptoms occurs in later stages of bvFTD. Negative symptoms, such as apathy, aspontaneity, self-neglect, tend to increase and might overshadow positive symptoms and stereotypy as the disease progresses. Negative symptoms decreased in psychiatric cases, potentially related to treatment. Previous studies have shown that increased $\mathrm{FBI}$ positive symptoms at the initial visit have a relatively high potential to discriminate bvFTD from psychiatric diagnoses,(Boutoleau-Bretonniere et al., 2012; Dols et al., 2016; Krudop et al., 2015). Conversely, the distinctive trajectory for the FBI in bVFTD, psychiatric and other neurodegenerative disorders was predominantly driven by negative symptoms rather than positive symptoms. Our research group has previously 
observed high levels of stereotypy at baseline in bvFTD patients,(Krudop et al., 2015; Vijverberg, et al., 2017). Although another study observed that stereotyped behaviours decrease over time in bvFTD patients, we found that these symptoms remain high and do not change over time(O'Connor et al., 2016).

Decline on the MMSE was similar in bVFTD and other neurodegenerative disorders, whereas a faster decline on the FAB was observed in bvFTD patients. Our results are in line with some,(Ramanan et al., 2017; Smits et al., 2015), but not all(Tan et al., 2013), studies who found only moderate discriminative value between bvFTD and other neurodegenerative disorders on longitudinal trajectories for cognition. Further research is needed to identify whether the progression of other neuropsychological measures can differentiate between bvFTD and its differential diagnosis reliably.

At baseline, bvFTD patients performed worse on emotion recognition (Ekman) compared to psychiatric patients (Gossink et al., 2017). Contrary to our expectations, none of the groups showed change in social cognitive functioning over time. Our findings are in contrast to those reported by Kumfor et al., (2014), who observed a more rapid decline on emotion recognition in bvFTD compared to other neurodegenerative disorders(Kumfor et al., 2014). One explanation for this discrepancy could be that patients from our study showed lower emotion recognition performances at baseline, leaving less space for decline(Kumfor et al., 2014). Theory of mind performances, as estimated with the Faux pas test, were initially similar between disorders, and worsened over time in all diagnostic groups(Gossink et al., 2017). Performances on empathy, however, did not change over time, suggesting that Faux pas improvements were not driven by improvements in theory of mind specifically. Our results suggest that current methods for measuring social cognition are not sensitive for 
measuring disease progression. Considering that social cognitive decline is one of the first symptoms of bvFTD, it is conceivable that social cognition was already so much affected in our cohort that further progression cannot be detected(Rascovsky et al., 2011). Consequently, monitoring social cognitive functioning might, at least for Ekman Faces and Faux Pas tests, not be useful for tracking disease progression.

We expected more neuronal damage, as estimated with atrophy on neuroimaging and CSF biomarkers, to be associated with disease progression. When studying these clinicopathological relationships, we observed that smaller caudate volumes were nominally associated with a faster worsening on stereotyped behaviour (SRI) over time across all diagnostic groups. Previous neuroimaging studies have implied the involvement of the caudate in the pathology of stereotyped behaviour, as its part of cortico-striato-thalamocortico circuits(Milad and Rauch, 2012).

We further observed that high levels of axonal damage, as reflected by high CSF NfL levels, were associated with decline on the FAB,(Meeter et al., 2016; Pijnenburg et al., 2015). Higher CSF NfL levels have earlier been associated with FTD(Meeter et al., 2016). Nevertheless, higher CSF NfL levels have also been observed in other neurodegenerative disorders, and to a lesser extent in psychiatric disorders(Vijverberg, et al., 2017)(Table 1). Altogether, this suggests that higher CSF NfL levels are not specifically associated with disease progression in bvFTD, but also in psychiatric and other neurodegenerative disorders. Limitations of this study include that the diagnostic workup took disease progression of the patient into account, whereas at the same time we examined the longitudinal relationship between diagnoses and several measures on social cognition, cognition and behaviour. Nevertheless, diagnoses in this study were based upon the composite of the diagnostic 
work-up, rather than questionnaires only,(Krudop et al., 2014; Van der Flier, 2014). Second, behaviour and social cognition are complex constructs making them in general challenging to measure objectively. Moreover, our results could have been confounded by a high dropoutrate in bvFTD patients, although no association was observed between clinical measures and dropout and follow-up duration was not lower in bvFTD patients. Additionally, although diagnoses were based on a two year follow-up period, we cannot exclude the possibility that our dataset comprises misdiagnoses as no golden standard exists for bvFTD diagnosis during life. However, the definite bvFTD patients included in this study had a genetic mutation related to FTD. Another relevant limitation of this study is that MRI scans were acquired on different scanner types ( $n=45$ GE and $n=8$ Phillips, both 3T) and only collected in a subgroup ( $n=47$ after quality check FreeSurfer output) of all patients. This could have biased our study, as scanner type may influence estimated brain volume and patients not willing to undergo MR scanning may have different clinical characteristics than those who are.

Although we were able to detect differences between groups in disease progression for clinical measures (FBI, FAB and MMSE), it should be acknowledged that diagnostic groups in the current study were heterogeneous, because of their behaviour-based inclusion. Within diagnostic groups some heterogeneity may exist in disease progression. Specifically, schizophrenia and bipolar disorder have been associated with mild cognitive impairment or dementia(Samame et al., 2014; Schouws et al., 2016). However, we believe that - at least for schizophrenia - this had no significant influence on our results as our study sample comprised only one schizophrenia patient. Also, we do not expect that the bipolar disorder patients in this study $(n=7)$ have a faster disease progression than other psychiatric cases, as no differences have been reported in cognitive decline between bipolar disorder patients and healthy elderly(Samame et al., 2014; Schouws et al., 2016). Finally, as inclusion of our 
patients was symptom based, it is impossible to draw conclusions on specific diagnostic groups within the psychiatric subgroup of patients and within the group of patients with another neurodegenerative disease. At the same time, we are convinced that this symptombased design is a strength of the current study as it resembles clinical practice.

A strength of this study is that diagnosis took place with both a neurologist, psychiatrist and neuropsychologist, minimizing the chance on misdiagnoses. Additionally, the sample size of the current study was relatively high compared to former longitudinal studies on social cognition or behaviour in bvFTD,(Boutoleau-Bretonniere et al., 2012; Kumfor et al., 2014; O'Connor et al., 2016). Another strength of our study is the use of behavioural and social cognitive instruments, whereas other longitudinal studies in bvFTD have mainly used general dementia rating instruments, such as the MMSE and Clinical Dementia Rating (CDR), thereby only appreciating cognitive decline in FTD - while social cognitive and behavioural abnormalities are core features of bvFTD,(Josephs et al., 2011; Smits et al., 2015).

In summary, this study demonstrates that tracking frontal behavioural symptoms and cognition over time might aid in differentiating bvFTD from psychiatric and other neurodegenerative disorders. Tracking stereotypy and social cognition may be less informative in differentiating between these disorders, although the presence of increased stereotyped behaviour and decreased social cognition at baseline are suggestive of bvFTD. Baseline MRI and/or CSF NfL values did not seem to have clinical relevance in predicting disease course, but do implicate a pathophysiological role for CSF NfL levels in the progression of frontal lobe dysfunction. 


\section{TABLES AND FIGURES}

Table 1. Demographic and clinical baseline characteristics.

\begin{tabular}{|c|c|c|c|c|}
\hline & bvFTD & Psychiatric diagnosis & Neurodegenerative diagnosis & Between-group comparisons \\
\hline "Gender (female/male) ${ }^{c}$ & $15 / 19$ & $9 / 34$ & 9/19 & $\chi^{2}{ }_{2}=4.746, p=0.093$ \\
\hline Age (years) ${ }^{a, c}$ & $63.2(6.7)$ & $59.8(7.0)$ & $64.5(6.4)$ & $F_{2,104}=4.675, p=\mathbf{0 . 0 1 1}$ \\
\hline Disease duration (years) ${ }^{b, c}$ & $3.0(2.0-7.0)$ & $3.0(2.0-5.0)$ & $3.0(2.0-4.0)$ & $H_{2}=1.096, p=0.579$ \\
\hline Follow-up time (years) ${ }^{b, c}$ & $1.5(0.0-2.0)$ & $1.9(1.2-2.2)$ & $1.9(1.1-2.0)$ & $\mathrm{H}_{2}=3.466, \mathrm{p}=0.178$ \\
\hline CSF NfL $^{b, d}$ & $2089.0(939.6-3946.0)$ & $664.4(522.5$ - 943.7) & $1027.2(647.9-1750.7)$ & $\mathrm{H}_{2}=19.598, p=5.6 \times 10^{-5}$ \\
\hline CSF p/t-tau ${ }^{a, d}$ & $0.12(0.04)$ & $0.17(0.04)$ & $0.15(0.03)$ & $F_{2,76}=16.372, p=1.0 \times 10^{-6}$ \\
\hline CSF YKL-40 ${ }^{b, d}$ & $347.6(250.5-479.5)$ & $233.8(171.1-301.5)$ & $283.4(228.4-322.7)$ & $\mathrm{H}_{2}=11.725, \mathbf{p}=\mathbf{0 . 0 0 3}$ \\
\hline Total GM volume $\left(\mathrm{mm}^{3}\right)$ & $5702760(72423)$ & $580740(68142)$ & $571262(56594)$ & $F_{2,54}=0.158, p=0.854$ \\
\hline NBV & $0.37(0.024)$ & $0.38(0.040)$ & $0.37(0.027)$ & $F_{2,54}=0.414, p=0.663$ \\
\hline Fazekas score & $13 / 14 / 5 / 0$ & $24 / 13 / 3 / 0$ & $12 / 11 / 2 / 1$ & $X^{2}=6.272, p=0.393$ \\
\hline FBI ${ }^{a, c}$ & $26.3(9.7)$ & $26.0(8.4)$ & $24.2(9.6)$ & $F_{2,102}=0.462, p=0.631$ \\
\hline \# no. $F B I^{b, f}$ & $2.0(1.0-3.0)$ & $3.0(2.0-3.0)$ & $3.0(2.0-3.0)$ & $\mathrm{H}_{2}=8.784, \mathbf{p}=\mathbf{0 . 0 1 2}$ \\
\hline
\end{tabular}




\section{\# no. MMSE ${ }^{\text {b,h }}$}

$$
1.5(1.0-2.0)
$$

\section{$F A B^{b, e}$}

$$
16.0(13.5-17.5)
$$

$$
16.0(13.8-18.0)
$$

$$
2.0(1.0-2.5)
$$

$$
3.0(2.0-3.0)
$$

\section{Faux pas total correct ${ }^{a, e}$}

\section{\# no. Faux Pas total ${ }^{b, j}$}

\section{Ekman $60^{\text {a,e }}$}

\# no. Ekman $60^{\mathrm{b}, \mathrm{k}}$

$$
6.1(2.7)
$$

$$
1.0(0.0-1.0)
$$

$$
1.0(1.0-1.0)
$$

$$
7.4(2.0)
$$

$$
1.0(1.0-2.0)
$$

$$
1.0(1.0-2.0)
$$

$$
6.5(2.3)
$$$$
2.0(1.0-2.0)
$$

$2.0(1.0-2.0)$
$\mathrm{H}_{2}=4.034, p=0.133$

$H_{2}=22.255, p=1.5 \times 10^{-5}$

$F_{2,65}=2.229, p=0.116$

$H_{2}=11.067, p=0.004$

$F_{2,82}=7.194, p=0.001$

$H_{2}=17.406, p=1.7 \times 10^{-4}$

bvFTD: behavioural variant frontotemporal dementia, CSF: cerebrospinal fluid, NfL: neurofilament light, p-tau: , t-tau: total tau, YKL-40: chitinase-3-like protein 1, FBI: frontal behavioural inventory, SRI: the stereotypy rating inventory, MMSE: mini-mental state examination, FAB: frontal assessment battery, \#no.: number of measurements, NBV: normalized brain volume, GM: grey matter. Significant associations $(p<0.05)$ are depicted in bold

a Mean (standard deviation). ${ }^{\mathrm{b}}$ Median (first quartile - third quartile).

${ }^{c} n=34$ bvFTD, $n=43$ psychiatric diagnosis and $n=28$ neurodegenerative disorder patients.

${ }^{d}$ NfL sample size: $n=17$ bvFTD, $n=25$ psychiatric diagnosis and $n=18$ neurodegenerative disorder patients. P/t-tau: $n=28$ bvFTD, $n=29$ psychiatric diagnosis and $n=20$ neurodegenerative disorder patients. YKL-40 sample size: $\mathrm{n}=20$ bvFTD, $\mathrm{n}=25$ psychiatric diagnosis, and $\mathrm{n}=18$ neurodegenerative disorder patients.

e SRI sample size: $n=34$ bvFTD, $n=43$ psychiatric diagnosis and $n=26$ neurodegenerative disorder patients, FAB sample size: $n=33$ bvFTD, $n=42$ psychiatric diagnosis and $n=27$ neurodegenerative disorder patients, Faux pas sample size: $n=22$ bvFTD, $n=25$ psychiatric diagnosis and $n=21$ neurodegenerative disorder patients, Ekman sample size: $n=27$ bvFTD, $n=31$ psychiatric diagnosis and $n=27$ neurodegenerative disorder patients. ${ }^{\mathrm{f}} \mathrm{FBI}$ : bvFTD versus psychiatry: $\mathrm{U}=548.500, \mathrm{p}=0.041$, bvFTD versus neurodegenerative: $\mathrm{U}=295.000, \mathrm{p}=0.005$. 
g SRI bvFTD versus psychiatry: $\mathrm{U}=537.000, \mathrm{p}=0.029$, bvFTD versus neurodegenerative: $\mathrm{U}=312.000, \mathrm{p}=0.011$.

${ }^{\mathrm{h}}$ MMSE bvFTD versus psychiatry: $\mathrm{U}=394.500, \mathrm{p}=2.097 \times 10^{-4}$, bvFTD versus neurodegenerative: $\mathrm{U}=193.000, \mathrm{p}=1.800 \times 10^{-5}$

i FAB bvFTD versus psychiatry: $\mathrm{U}=390.500, \mathrm{p}=1.870 \times 10^{-4}$, bvFTD versus neurodegenerative: $\mathrm{U}=197.000, \mathrm{p}=2.600 \times 10^{-5}$.

jFaux Pas total bvFTD versus psychiatry: $U=522.000, p=0.022$, bvFTD versus neurodegenerative: $U=263.000, p=0.001$

k Ekman 60 bvFTD versus psychiatry: $\mathrm{U}=520.000, \mathrm{p}=0.017$, bvFTD versus neurodegenerative: $\mathrm{U}=205.500, \mathrm{p}=2.400 \times 10^{-5}$, psychiatry versus neurodegenerative: $\mathrm{U}=446.500, \mathrm{p}=0.040$. 
Table 2. Progression of behaviour, cognition and social cognition across and between diagnosis.

\begin{tabular}{|c|c|c|c|c|c|c|c|c|}
\hline & \multicolumn{2}{|c|}{ Model 1} & \multicolumn{6}{|c|}{ Model 2} \\
\hline & \multicolumn{2}{|c|}{ Time } & \multicolumn{2}{|c|}{ Diagnosis * time interaction } & \multicolumn{2}{|c|}{ Time } & \multicolumn{2}{|c|}{ Diagnosis * time interaction } \\
\hline & $\mathbf{F}$ & p value & $\mathbf{F}$ & p value & $\mathbf{F}$ & p value & $\mathbf{F}$ & p value \\
\hline$\overline{\text { FBI }}$ & 0.341 & 0.561 & 8.513 & $4.3 \times 10^{-4^{* * *}}$ & 0.308 & 0.580 & 8.357 & $4.9 \times 10^{-4^{* * *}}$ \\
\hline FBI positive subscale & 0.327 & 0.569 & 2.370 & 0.099 & 0.388 & 0.535 & 2.294 & 0.107 \\
\hline FBI negative subscale & 0.426 & 0.516 & 9.557 & $1.9 \times 10^{-4^{* * *}}$ & 0.312 & 0.578 & 9.279 & $2.4 \times 10^{-3 * *}$ \\
\hline SRI & 0.378 & 0.541 & 1.627 & 0.204 & 0.495 & 0.484 & 1.531 & 0.223 \\
\hline MMSE & 11.616 & $0.001 * *$ & 4.547 & $0.014^{*}$ & 11.438 & $0.001 * *$ & 4.201 & $0.019 *$ \\
\hline FAB & 6.915 & $0.010^{*}$ & 7.020 & $0.002 *$ & 7.128 & $0.009 * *$ & 6.903 & $0.002 * *$ \\
\hline Faux pas total correct & 3.935 & 0.055 & 0.044 & 0.957 & 4.443 & $0.042 *$ & 0.021 & 0.980 \\
\hline Faux pas correct & 5.465 & $0.023 *$ & 0.523 & 0.596 & 7.029 & $0.009 * *$ & 0.712 & 0.494 \\
\hline Non faux pas correct & 0.258 & 0.615 & 0.680 & 0.513 & 0.232 & 0.633 & 0.649 & 0.528 \\
\hline Empathy correct & 0.035 & 0.851 & 0.469 & 0.628 & 0.259 & 0.612 & 0.629 & 0.535 \\
\hline Ekman 60 & 0.224 & 0.638 & 0.833 & 0.440 & 0.135 & 0.715 & 0.695 & 0.504 \\
\hline
\end{tabular}

bvFTD: behavioural variant frontotemporal dementia, FBI: frontal behavioural inventory, SRI: stereotypical rating inventory, MMSE: mini-mental state examination, FAB: frontal assessment battery. Results of model 1 are generated excluding, and results of model 2 are generated including age, gender and education (years) as fixed baseline predictors. Significant associations are depicted in bold. ${ }^{* *}$ depicts a significance of $p<0.0001, * * p<0.01$ and $* p<0.05$ 
Table 3. Progression of behaviour, cognition and social cognition separately for diagnostic groups.

\begin{tabular}{|c|c|c|c|c|c|c|}
\hline & \multicolumn{2}{|c|}{ bvFTD } & \multicolumn{2}{|c|}{ Psychiatric diagnosis } & \multicolumn{2}{|c|}{ Neurodegenerative diagnosis } \\
\hline & Model 1 & Model 2 & Model 1 & Model 2 & Model 1 & Model 2 \\
\hline & B (SE) & B (SE) & B (SE) & B (SE) & B (SE) & B (SE) \\
\hline FBI & $2.902(1.124)^{*}$ & 2.917 (1.249)* & -3.536 $(0.956)^{* * *}$ & -3.494 (0.961)** & (-0.526(1.218) & -0.529(1.221) \\
\hline FBI positive subscale & $0.512(0.567)$ & $0.495(0.570)$ & $1.489(0.710)^{*}$ & $-0.987(0.434)^{*}$ & $-0.051(0.560)$ & $-0.076(0.563)$ \\
\hline FBI negative subscale & $2.253(0.873)^{*}$ & $2.286(0.880)^{*}$ & $-2.561(0.672)^{* *}$ & $-2.496(0.678)^{* *}$ & $-0.604(0.859)$ & $-0.576(0.863)$ \\
\hline SRI & $-0.885(0.979)$ & $-0.321(1.031)$ & $-0.533(0.829)$ & $-0.315(0.806)$ & $1.819(1.066)$ & $1.810(1.037)$ \\
\hline MMSE & $-1.538(0.540)^{* *}$ & $-1.571(0.556)^{* *}$ & $0.095(0.332)$ & $0.073(0.351)$ & $-1.144(0.417)^{* *}$ & $-1.116(0.430)^{* *}$ \\
\hline FAB & $-1.624(0.448)^{* * *}$ & $-1.663(0.454)^{* * *}$ & $0.312(0.262)$ & $0.296(0.270)$ & $-0.315(0.337)$ & $-0.311(0.341)$ \\
\hline Faux pas total correct & $0.236(0.380)$ & $0.298(0.377)$ & 0.338 (0.187)\# & $0.322(0.185) \#$ & $0.365(0.211)$ & $0.370(0.210)$ \\
\hline Faux pas correct & $0.204(0.344)$ & $0.182(0.291)$ & $0.287(0.171)$ & $0.79(0.141)$ & $0.526(0.205)^{*}$ & $0.502(0.165)^{* *}$ \\
\hline Non faux pas correct & $0.302(0.302)$ & $0.293(0.301)$ & $-0.008(0.149)$ & $-0.009(0.149)$ & $-0.102(0.172)$ & $-0.102(0.171)$ \\
\hline Empathy correct & $0.048(0.426)$ & $0.254(0.359)$ & $-0.138(0.223)$ & $-0.129(0.183)$ & $0.193(0.261)$ & $0.106(0.210)$ \\
\hline Ekman 60 & $-0.894(1.215)$ & $-0.798(1.258)$ & $0.596(0.684)$ & $0.589(0.693)$ & $-0.447(0.737)$ & $-0.386(0.751)$ \\
\hline
\end{tabular}


bVFTD: behavioural variant frontotemporal dementia, FBI: frontal behavioural inventory, SRI: stereotypical rating inventory, MMSE: mini-mental state examination, FAB: frontal assessment battery, SE: standard error. Results of model 1 are generated excluding, and results of model 2 are generated including age, gender and education (years) as fixed baseline predictors. B estimate coefficients indicate the change in cognitive measure per year, as estimated by model 1 and model 2 . Significant associations are depicted in bold. ${ }^{* * *}$ depicts a significance of $p<0.0001,{ }^{* *} p<0.001,{ }^{*} p<0.05$ and $\# p<0.10$.

Table 4. Association of baseline subcortical volumes with progression of behaviour and cognition over time.

\begin{tabular}{|c|c|c|c|c|c|c|c|c|c|c|c|c|}
\hline \multirow[b]{2}{*}{ Interaction } & \multicolumn{3}{|c|}{ MMSE } & \multicolumn{3}{|c|}{ FAB } & \multicolumn{3}{|c|}{ FBI } & \multicolumn{3}{|c|}{ SRI } \\
\hline & & & & & & & & & & & & \\
\hline time & B (SE) & p uncorr & p-FDR & B (SE) & p uncorr & p-FDR & B (SE) & p uncorr & p-FDR & B (SE) & p uncorr & p-FDR \\
\hline Thalamus & $0.000(0.000)$ & 0.742 & 0.982 & $0.000(0.000)$ & 0.534 & 0.745 & $-0.001(0.001)$ & 0.383 & 0.936 & $-0.000(0.001)$ & 0.662 & 0.772 \\
\hline Caudate & $0.000(0.001)$ & 0.860 & 0.982 & $0.000(0.000)$ & 0.638 & 0.745 & $-0.000(0.001)$ & 0.874 & 0.936 & $0.004(0.002)$ & 0.059 & 0.413 \\
\hline Putamen & $0.000(0.001)$ & 0.637 & 0.982 & $0.000(0.000)$ & 0.745 & 0.745 & $0.000(0.001)$ & 0.936 & 0.936 & $0.001(0.001)$ & 0.387 & 0.715 \\
\hline Pallidum & $0.000(0.001)$ & 0.886 & 0.982 & $0.000(0.001)$ & 0.694 & 0.745 & $-0.001(0.003)$ & 0.632 & 0.936 & $0.003(0.004)$ & 0.354 & 0.715 \\
\hline Hippocampus & $-0.000(0.000)$ & 0.982 & 0.982 & $-0.000(0.000)$ & 0.517 & 0.745 & $0.001(0.001)$ & 0.675 & 0.936 & $0.000(0.002)$ & 0.791 & 0.791 \\
\hline Amygdala & $0.000(0.001)$ & 0.737 & 0.982 & $-0.000(0.001)$ & 0.680 & 0.745 & $-0.000(0.002)$ & 0.844 & 0.936 & $0.002(0.003)$ & 0.503 & 0.715 \\
\hline NAcc & $0.001(0.001)$ & 0.334 & 0.982 & $0.002(0.001)$ & 0.299 & 0.745 & $-0.002(0.006)$ & 0.693 & 0.936 & $0.005(0.007)$ & 0.511 & 0.715 \\
\hline
\end{tabular}

ICV: intracranial volume, FBI: frontal behavioural inventory, SRI: stereotypical rating inventory, MMSE: mini-mental state examination, FAB: frontal assessment battery, uncorr: uncorrected, FDR: false-discovery rate, SE: standard error, NAcc: nucleus accumbens. 
Table 5. Association of baseline cortical thickness with progression of behaviour and cognition over time.

\begin{tabular}{|c|c|c|c|c|c|c|c|c|c|c|c|c|}
\hline \multirow[b]{2}{*}{ Interaction time } & \multicolumn{3}{|c|}{ MMSE } & \multicolumn{3}{|c|}{ FAB } & \multicolumn{3}{|c|}{ FBI } & \multicolumn{3}{|c|}{ SRI } \\
\hline & B (SE) & p uncorr & p-FDR & B (SE) & p uncorr & p-FDR & B (SE) & p uncorr & p-FDR & B (SE) & p uncorr & p-FDR \\
\hline Frontal pole & $0.350(0.537)$ & 0.515 & 0.986 & $0.433(0.555)$ & 0.436 & 0.948 & $0.087(1.977)$ & 0.965 & 0.989 & $-4.166(2.587)$ & 0.110 & 0.667 \\
\hline Lateralorbitfrontal & $0.587(0.954)$ & 0.539 & 0.986 & $0.417(0.970)$ & 0.668 & 0.948 & $0.471(3.418)$ & 0.891 & 0.989 & $-3.194(4.512)$ & 0.480 & 0.934 \\
\hline Medialorbitofrontal & $0.372(0.903)$ & 0.681 & 0.986 & $0.341(0.923)$ & 0.712 & 0.948 & $0.700(3.075)$ & 0.820 & 0.989 & $-3.708(3.832)$ & 0.335 & 0.871 \\
\hline Rostralmiddlefrontal & $0.584(1.421)$ & 0.681 & 0.986 & $1.415(1.443)$ & 0.328 & 0.948 & $0.884(6.260)$ & 0.888 & 0.989 & $-12.282(6.961)$ & 0.081 & 0.667 \\
\hline Superiorfrontal & $1.166(1.780)$ & 0.513 & 0.986. & $1.657(1.765)$ & 0.349 & 0.948 & $-2.953(7.092)$ & 0.678 & 0.989 & $-10.397(7.215)$ & 0.154 & 0.667 \\
\hline Entorhinal & $0.018(0.338)$ & 0.957 & 0.986 & $-0.342(0.346)$ & 0.325 & 0.948 & $0.334(0.970)$ & 0.732 & 0.989 & 1.212 (1.247) & 0.333 & 0.871 \\
\hline Fusiform & $0.228(1.106)$ & 0.830 & 0.986 & $-0.603(1.076)$ & 0.576 & 0.948 & $0.359(3.892)$ & 0.927 & 0.989 & $1.745(4.423)$ & 0.694 & 0.934 \\
\hline Inferiortemporal & $0.204(0.884)$ & 0.818 & 0.986 & 0.165 (0.899) & 0.854 & 0.948 & $-0.296(3.471)$ & 0.932 & 0.989 & $-0.344(4.167)$ & 0.934 & 0.934 \\
\hline Insula & $-0.046(0.883)$ & 0.959 & 0.986 & $-0.567(0.911)$ & 0.534 & 0.948 & $0.418(3.353)$ & 0.901 & 0.989 & 0.721 (4.319) & 0.868 & 0.934 \\
\hline Middletemporal & $0.111(0.974)$ & 0.910 & 0.986 & $-0.002(0.993)$ & 0.998 & 0.998 & $-0.178(3.462)$ & 0.959 & 0.989 & $-1.836(4.162)$ & 0.660 & 0.934 \\
\hline Parahippocampal & $0.097(0.467)$ & 0.836 & 0.986 & $0.155(0.476)$ & 0.746 & 0.948 & $0.890(1.881)$ & 0.637 & 0.989 & $0.260(2.270)$ & 0.909 & 0.934 \\
\hline Superiortemporal & $0.276(1.002)$ & 0.783 & 0.986. & $-0.162(1.027)$ & 0.875 & 0.948 & $0.047(3.405)$ & 0.989 & 0.989 & $0.548(3.926)$ & 0.889 & 0.934 \\
\hline Temporal pole & $0.006(0.352)$ & 0.986 & 0.986 & $-0.319(0.362)$ & 0.379 & 0.948 & $0.124(1.425)$ & 0.931 & 0.989 & $0.294(1.766)$ & 0.868 & 0.934 \\
\hline
\end{tabular}

standard error. 
Table 6. Association of baseline CSF with progression of behaviour and cognition over time.

\begin{tabular}{|c|c|c|c|c|c|c|c|c|}
\hline \multirow{2}{*}{ Interaction } & \multicolumn{2}{|c|}{ MMSE } & \multicolumn{2}{|c|}{ FAB } & \multicolumn{2}{|l|}{ FBI } & \multicolumn{2}{|c|}{ SRI } \\
\hline & $B(S E)$ & $\mathbf{p}$ & $B(S E)$ & p & $B(S E)$ & p & B (SE) & $\mathbf{p}$ \\
\hline CSF NfL & $-0.000(0.000)$ & 0.240 & $-0.001(0.000)$ & $0.038^{*}$ & $-0.000(0.001)$ & 0.744 & $0.000(0.001)$ & 0.934 \\
\hline CSF p/t-tau & $7.410(8.803)$ & 0.403 & 8.631 (7.774) & 0.271 & $-27.190(24.142)$ & 0.265 & $-9.626(19.603)$ & 0.625 \\
\hline CSF YKL-40 & $-0.002(0.003)$ & 0.353 & $-0.002(0.002)$ & 0.298 & $-0.009(0.008)$ & 0.288 & $-0.002(0.006)$ & 0.744 \\
\hline
\end{tabular}

CSF: cerebrospinal fluid, NfL: neurofilament light chain, t-tau: total tau, p-tau: phosphotau ${ }_{181}$, YKL-40: chitinase-3-like protein, FBI: frontal behavioural inventory, SRI: stereotypical rating inventory, MMSE: minimental state examination, FAB: frontal assessment battery, SE: standard error. Interaction is displayed for baseline CSF protein level* clinical measure*time. Significant associations are depicted in bold. *depicts a significance of $p<0.05$. 

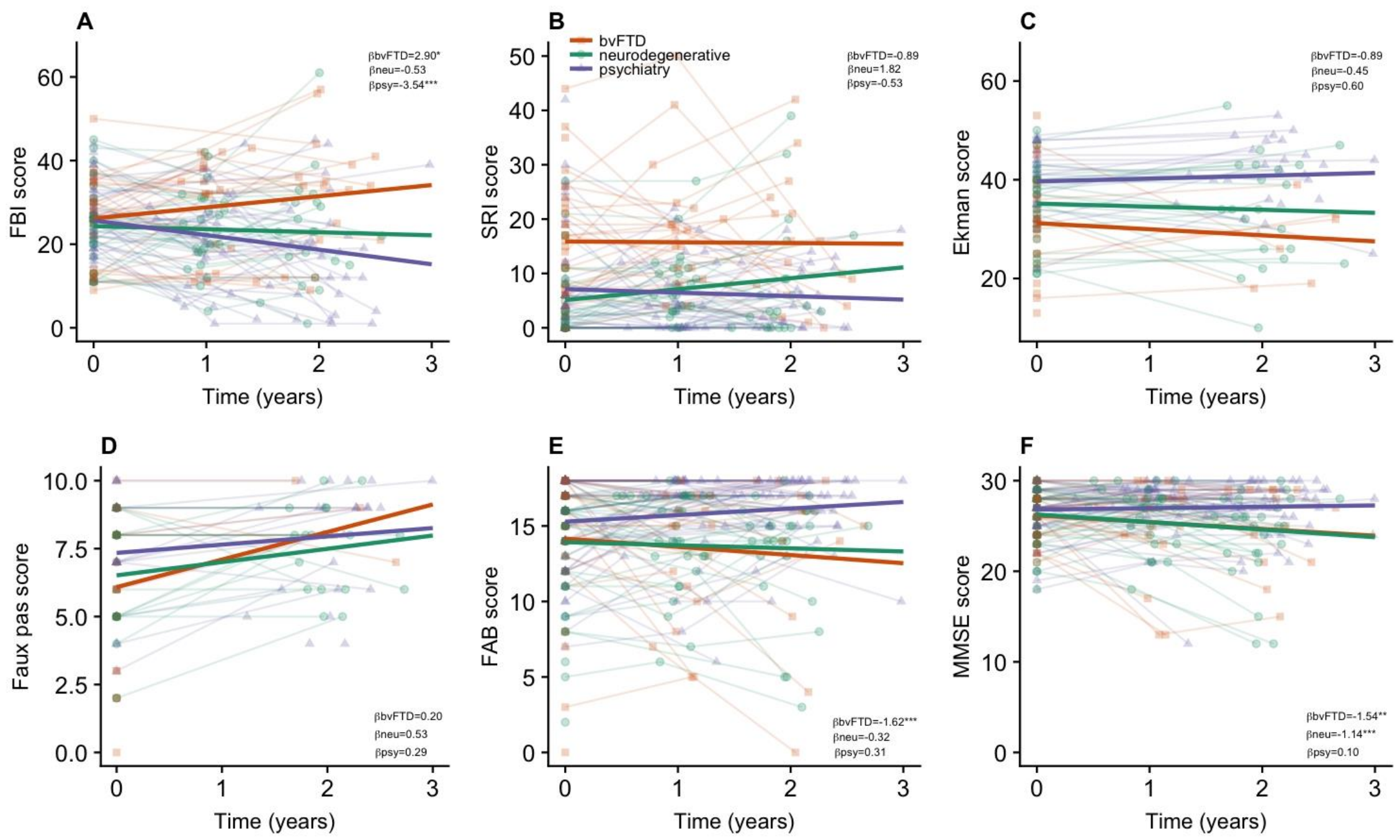
Figure 1. Change in behaviour, social cognitive and cognitive measures according to diagnosis. bvFTD: behavioural variant frontotemporal dementia, Neu.:

neurodegenerative, psy: psychiatric, FBI: frontal behavioural inventory, SRI: stereotypical rating inventory, FAB: frontal assessment battery, MMSE: mini-mental state examination. Regression lines are created by applying model 1 to each group. Results of model 1 are generated excluding age, gender and education (years) as fixed baseline predictors. $\beta$ estimate coefficients indicate the change in cognitive measure per year, as estimated by model $1 .{ }^{* *}$ depicts a significance of $\mathrm{p}<0.0001,{ }^{* *} \mathrm{p}<0.001$ and ${ }^{*} \mathrm{p}<0.05$. MMSE range $=0-30, \mathrm{FAB}$ range $=0-18$, with lower scores reflecting more severe cognitive impairment. FBI range $=0-72$, SRI range $=0-60$, with higher scores reflecting more severe behavioural impairment. Ekman range=0-60, with higher scores reflecting better performances. Faux pas total correct=Faux Pas correct + Non Faux Pas correct, range=0-10, with higher scores reflecting better performances. Total number of measurements is 249 for FBI , 248 for SRI, 139 for Ekman, 116 for Faux pas, 233 for FAB and 237 for MMSE. 


\section{REFERENCES}

Andrew, K., Neelesh, Nadkarni, Wilda, Davidson, \& Alexw, Thomas, 2000. The frontal behavioural inventory in the differential diagnosis of frontotemporal dementia. Journal of the International Neuropsychological Society 6, 460-468.

American Psychiatric Association, 2000. Diagnostic criteria from DSM-IV. American Psychiatric Pub. Baron-Cohen, S., O'Riordan, M., Stone, V., Jones, R., Plaisted, K., 1999. Recognition of faux pas by normally developing children and children with Asperger syndrome or high-functioning autism. J Autism Dev Disord 29(5), 407-418.

Borroni, B., Grassi, M., Premi, E., Alberici, A., Cosseddu, M., Cancelli, V., Caobelli, F., Paghera, B., Padovani, A., 2012. Is long-term prognosis of frontotemporal lobar degeneration predictable by neuroimaging? Evidence from a single-subject functional brain study. J Alzheimers Dis 29(4), 883-890. Boutoleau-Bretonniere, C., Lebouvier, T., Volteau, C., Jaulin, P., Lacomblez, L., Damier, P., ThomasAnterion, C., Vercelletto, M., 2012. Prospective evaluation of behavioral scales in the behavioral variant of frontotemporal dementia. Dement Geriatr Cogn Disord 34(2), 75-82.

Desikan, R.S., Segonne, F., Fischl, B., Quinn, B.T., Dickerson, B.C., Blacker, D., Buckner, R.L., Dale, A.M., Maguire, R.P., Hyman, B.T., Albert, M.S., Killiany, R.J., 2006. An automated labeling system for subdividing the human cerebral cortex on MRI scans into gyral based regions of interest. Neuroimage 31(3), 968-980.

Diehl-Schmid, J., Pohl, C., Ruprecht, C., Wagenpfeil, S., Foerstl, H., Kurz, A., 2007. The Ekman 60 Faces Test as a diagnostic instrument in frontotemporal dementia. Arch Clin Neuropsychol 22(4), 459-464. Dolan, R.J., 1999. On the neurology of morals. Nat Neurosci 2(11), 927-929.

Dols, A., van Liempt, S., Gossink, F., Krudop, W.A., Sikkes, S., Pijnenburg, Y.A., Stek, M.L., 2016. Identifying Specific Clinical Symptoms of Behavioral Variant Frontotemporal Dementia Versus Differential Psychiatric Disorders in Patients Presenting With a Late-Onset Frontal Lobe Syndrome. J Clin Psychiatry 77(10), 1391-1395.

Dubois, B., Slachevsky, A., Litvan, I., Pillon, B., 2000. The FAB: a Frontal Assessment Battery at bedside. Neurology 55(11), 1621-1626.

Fischl, B., Dale, A.M., 2000. Measuring the thickness of the human cerebral cortex from magnetic resonance images. Proc Natl Acad Sci U S A 97(20), 11050-11055.

Fischl, B., van der Kouwe, A., Destrieux, C., Halgren, E., Segonne, F., Salat, D.H., Busa, E., Seidman, L.J., Goldstein, J., Kennedy, D., Caviness, V., Makris, N., Rosen, B., Dale, A.M., 2004. Automatically parcellating the human cerebral cortex. Cereb Cortex 14(1), 11-22.

Folstein, M.F., Folstein, S.E., McHugh, P.R., 1975. "Mini-mental state". A practical method for grading the cognitive state of patients for the clinician. J Psychiatr Res 12(3), 189-198.

Galimberti, D., Fenoglio, C., Serpente, M., Villa, C., Bonsi, R., Arighi, A., Fumagalli, G.G., Del Bo, R., Bruni, A.C., Anfossi, M., Clodomiro, A., Cupidi, C., Nacmias, B., Sorbi, S., Piaceri, I., Bagnoli, S., Bessi, V., Marcone, A., Cerami, C., Cappa, S.F., Filippi, M., Agosta, F., Magnani, G., Comi, G., Franceschi, M., Rainero, I., Giordana, M.T., Rubino, E., Ferrero, P., Rogaeva, E., Xi, Z., Confaloni, A., Piscopo, P., Bruno, G., Talarico, G., Cagnin, A., Clerici, F., Dell'Osso, B., Comi, G.P., Altamura, A.C., Mariani, C., Scarpini, E., 2013. Autosomal dominant frontotemporal lobar degeneration due to the C9ORF72 hexanucleotide repeat expansion: late-onset psychotic clinical presentation. Biol Psychiatry 74(5), 384-391.

Gossink, F.T., Schouws, S., Krudop, W.A., Scheltens, P., Stek, M.L., Pijnenburg, Y., Dols, A., 2017. Social cognition differentiates behavioral variant frontotemporal dementia from other neurodegenerative diseases and psychiatric disorders Submitted.

Josephs, K.A., Jr., Whitwell, J.L., Weigand, S.D., Senjem, M.L., Boeve, B.F., Knopman, D.S., Smith, G.E., Ivnik, R.J., Jack, C.R., Jr., Petersen, R.C., 2011. Predicting functional decline in behavioural variant frontotemporal dementia. Brain 134(Pt 2), 432-448. 
Krudop, W.A., Kerssens, C.J., Dols, A., Prins, N.D., Moller, C., Schouws, S., Barkhof, F., van Berckel, B.N., Teunissen, C.E., van der Flier, W.M., Scheltens, P., Stek, M.L., Pijnenburg, Y.A., 2014. Building a new paradigm for the early recognition of behavioral variant frontotemporal dementia: Late Onset Frontal Lobe Syndrome study. Am J Geriatr Psychiatry 22(7), 735-740.

Krudop, W.A., Kerssens, C.J., Dols, A., Prins, N.D., Moller, C., Schouws, S., van der Flier, W.M., Scheltens, P., Sikkes, S., Stek, M.L., Pijnenburg, Y.A., 2015. Identifying bvFTD Within the Wide Spectrum of Late Onset Frontal Lobe Syndrome: A Clinical Approach. Am J Geriatr Psychiatry 23(10), 1056-1066.

Krudop, W.A., Pijnenburg, Y.A., 2015. Historical evolution of the frontal lobe syndrome. Psychopathology 48(4), 222-229.

Kumfor, F., Irish, M., Leyton, C., Miller, L., Lah, S., Devenney, E., Hodges, J.R., Piguet, O., 2014. Tracking the progression of social cognition in neurodegenerative disorders. J Neurol Neurosurg Psychiatry 85(10), 1076-1083.

McKeith, I.G., Dickson, D.W., Lowe, J., Emre, M., O'Brien, J.T., Feldman, H., Cummings, J., Duda, J.E., Lippa, C., Perry, E.K., Aarsland, D., Arai, H., Ballard, C.G., Boeve, B., Burn, D.J., Costa, D., Del Ser, T., Dubois, B., Galasko, D., Gauthier, S., Goetz, C.G., Gomez-Tortosa, E., Halliday, G., Hansen, L.A., Hardy, J., Iwatsubo, T., Kalaria, R.N., Kaufer, D., Kenny, R.A., Korczyn, A., Kosaka, K., Lee, V.M., Lees, A., Litvan, I., Londos, E., Lopez, O.L., Minoshima, S., Mizuno, Y., Molina, J.A., Mukaetova-Ladinska, E.B., Pasquier, F., Perry, R.H., Schulz, J.B., Trojanowski, J.Q., Yamada, M., Consortium on, D.L.B., 2005. Diagnosis and management of dementia with Lewy bodies: third report of the DLB Consortium. Neurology 65(12), 1863-1872.

McKhann, G.M., Knopman, D.S., Chertkow, H., Hyman, B.T., Jack, C.R., Jr., Kawas, C.H., Klunk, W.E., Koroshetz, W.J., Manly, J.J., Mayeux, R., Mohs, R.C., Morris, J.C., Rossor, M.N., Scheltens, P., Carrillo, M.C., Thies, B., Weintraub, S., Phelps, C.H., 2011. The diagnosis of dementia due to Alzheimer's disease: recommendations from the National Institute on Aging-Alzheimer's Association workgroups on diagnostic guidelines for Alzheimer's disease. Alzheimers Dement 7(3), 263-269.

Meeter, L.H., Dopper, E.G., Jiskoot, L.C., Sanchez-Valle, R., Graff, C., Benussi, L., Ghidoni, R., Pijnenburg, Y.A., Borroni, B., Galimberti, D., Laforce, R.J., Masellis, M., Vandenberghe, R., Ber, I.L., Otto, M., van Minkelen, R., Papma, J.M., Rombouts, S.A., Balasa, M., Oijerstedt, L., Jelic, V., Dick, K.M., Cash, D.M., Harding, S.R., Jorge Cardoso, M., Ourselin, S., Rossor, M.N., Padovani, A., Scarpini, E., Fenoglio, C., Tartaglia, M.C., Lamari, F., Barro, C., Kuhle, J., Rohrer, J.D., Teunissen, C.E., van Swieten, J.C., 2016. Neurofilament light chain: a biomarker for genetic frontotemporal dementia. Ann Clin Transl Neurol 3(8), 623-636.

Milad, M.R., Rauch, S.L., 2012. Obsessive-compulsive disorder: beyond segregated cortico-striatal pathways. Trends Cogn Sci 16(1), 43-51.

O'Connor, C.M., Clemson, L., Hornberger, M., Leyton, C.E., Hodges, J.R., Piguet, O., Mioshi, E., 2016. Longitudinal change in everyday function and behavioral symptoms in frontotemporal dementia. Neurol Clin Pract 6(5), 419-428.

Pijnenburg, Y.A., Verwey, N.A., van der Flier, W.M., Scheltens, P., Teunissen, C.E., 2015.

Discriminative and prognostic potential of cerebrospinal fluid phosphoTau/tau ratio and neurofilaments for frontotemporal dementia subtypes. Alzheimers Dement (Amst) 1(4), 505-512. Pose, M., Cetkovich, M., Gleichgerrcht, E., Ibanez, A., Torralva, T., Manes, F., 2013. The overlap of symptomatic dimensions between frontotemporal dementia and several psychiatric disorders that appear in late adulthood. Int Rev Psychiatry 25(2), 159-167.

Ramanan, S., Bertoux, M., Flanagan, E., Irish, M., Piguet, O., Hodges, J.R., Hornberger, M., 2017. Longitudinal Executive Function and Episodic Memory Profiles in Behavioral-Variant Frontotemporal Dementia and Alzheimer's Disease. J Int Neuropsychol Soc 23(1), 34-43.

Ranasinghe, K.G., Rankin, K.P., Pressman, P.S., Perry, D.C., Lobach, I.V., Seeley, W.W., Coppola, G., Karydas, A.M., Grinberg, L.T., Shany-Ur, T., Lee, S.E., Rabinovici, G.D., Rosen, H.J., Gorno-Tempini, M.L., Boxer, A.L., Miller, Z.A., Chiong, W., DeMay, M., Kramer, J.H., Possin, K.L., Sturm, V.E., Bettcher, B.M., Neylan, M., Zackey, D.D., Nguyen, L.A., Ketelle, R., Block, N., Wu, T.Q., Dallich, A., Russek, N., Caplan, A., Geschwind, D.H., Vossel, K.A., Miller, B.L., 2016. Distinct Subtypes of Behavioral Variant 
Frontotemporal Dementia Based on Patterns of Network Degeneration. JAMA Neurol 73(9), 10781088.

Rascovsky, K., Hodges, J.R., Knopman, D., Mendez, M.F., Kramer, J.H., Neuhaus, J., van Swieten, J.C., Seelaar, H., Dopper, E.G., Onyike, C.U., Hillis, A.E., Josephs, K.A., Boeve, B.F., Kertesz, A., Seeley, W.W., Rankin, K.P., Johnson, J.K., Gorno-Tempini, M.L., Rosen, H., Prioleau-Latham, C.E., Lee, A., Kipps, C.M., Lillo, P., Piguet, O., Rohrer, J.D., Rossor, M.N., Warren, J.D., Fox, N.C., Galasko, D., Salmon, D.P., Black, S.E., Mesulam, M., Weintraub, S., Dickerson, B.C., Diehl-Schmid, J., Pasquier, F., Deramecourt, V., Lebert, F., Pijnenburg, Y., Chow, T.W., Manes, F., Grafman, J., Cappa, S.F., Freedman, M., Grossman, M., Miller, B.L., 2011. Sensitivity of revised diagnostic criteria for the behavioural variant of frontotemporal dementia. Brain 134(Pt 9), 2456-2477.

Roman, G.C., Tatemichi, T.K., Erkinjuntti, T., Cummings, J.L., Masdeu, J.C., Garcia, J.H., Amaducci, L., Orgogozo, J.M., Brun, A., Hofman, A., et al., 1993. Vascular dementia: diagnostic criteria for research studies. Report of the NINDS-AIREN International Workshop. Neurology 43(2), 250-260.

Samame, C., Martino, D.J., Strejilevich, S.A., 2014. Longitudinal course of cognitive deficits in bipolar disorder: a meta-analytic study. J Affect Disord 164, 130-138.

Schouws, S.N., Comijs, H.C., Dols, A., Beekman, A.T., Stek, M.L., 2016. Five-year follow-up of cognitive impairment in older adults with bipolar disorder. Bipolar Disord 18(2), 148-154.

Segonne, F., Dale, A.M., Busa, E., Glessner, M., Salat, D., Hahn, H.K., Fischl, B., 2004. A hybrid approach to the skull stripping problem in MRI. Neuroimage 22(3), 1060-1075.

Segonne, F., Pacheco, J., Fischl, B., 2007. Geometrically accurate topology-correction of cortical surfaces using nonseparating loops. IEEE Trans Med Imaging 26(4), 518-529.

Shigenobu, K., Ikeda, M., Fukuhara, R., Maki, N., Hokoishi, K., Nebu, A., Yasuoka, T., Komori, K., Tanabe, H., 2002. The Stereotypy Rating Inventory for frontotemporal lobar degeneration. Psychiatry Res 110(2), 175-187.

Smits, L.L., van Harten, A.C., Pijnenburg, Y.A., Koedam, E.L., Bouwman, F.H., Sistermans, N., Reuling, I.E., Prins, N.D., Lemstra, A.W., Scheltens, P., van der Flier, W.M., 2015. Trajectories of cognitive decline in different types of dementia. Psychol Med 45(5), 1051-1059.

Tan, K.S., Libon, D.J., Rascovsky, K., Grossman, M., Xie, S.X., 2013. Differential longitudinal decline on the Mini-Mental State Examination in frontotemporal lobar degeneration and Alzheimer disease.

Alzheimer Dis Assoc Disord 27(4), 310-315.

Teunissen, C.E., Elias, N., Koel-Simmelink, M.J., Durieux-Lu, S., Malekzadeh, A., Pham, T.V., Piersma, S.R., Beccari, T., Meeter, L.H., Dopper, E.G., van Swieten, J.C., Jimenez, C.R., Pijnenburg, Y.A., 2016. Novel diagnostic cerebrospinal fluid biomarkers for pathologic subtypes of frontotemporal dementia identified by proteomics. Alzheimers Dement (Amst) 2, 86-94.

Teunissen, C.E., Petzold, A., Bennett, J.L., Berven, F.S., Brundin, L., Comabella, M., Franciotta, D., Frederiksen, J.L., Fleming, J.O., Furlan, R., Hintzen, R.Q., Hughes, S.G., Johnson, M.H., Krasulova, E., Kuhle, J., Magnone, M.C., Rajda, C., Rejdak, K., Schmidt, H.K., van Pesch, V., Waubant, E., Wolf, C., Giovannoni, G., Hemmer, B., Tumani, H., Deisenhammer, F., 2009. A consensus protocol for the standardization of cerebrospinal fluid collection and biobanking. Neurology 73(22), 1914-1922. Van der Flier, W.M.P., Y.A.L.; Prins, N.; Lemstra, A.W.; Bouwman, F.; Teunissen, C.E.; Van Berckel, B.N.M.; Stam, C.J.; Barkhof, F.; Visser, P.J.; Van Egmond, E.; Scheltens P., 2014. Optimizing Patient Care and Research: The Amsterdam Dementia Cohort. Journal of Alzheimer's Disease 41, 313-327. Vijverberg, E.G., Dols, A., Krudop, W.A., Del Campo Milan, M., Kerssens, C.J., Gossink, F., Prins, N.D., Stek, M.L., Scheltens, P., Teunissen, C.E., Pijnenburg, Y.A., 2017. Cerebrospinal fluid biomarker examination as a tool to discriminate behavioral variant frontotemporal dementia from primary psychiatric disorders. Alzheimers Dement (Amst) 7, 99-106.

Vijverberg, E.G.B., Gossink, F., Krudop, W.A., Sikkes, S., Kerssens, C.J., Prins, N.D., Stek, M.L., Scheltens, P., Pijnenburg, Y.A.L., Dols, A., 2017. The diagnostic challenge of the late-onset frontal lobe syndrome: Clinical predictors for primary psychiatric disorders versus behavioral variant frontotemporal dementia. Journal of Clinical Psychiatry, 1-18. 
Woolley, J.D., Khan, B.K., Murthy, N.K., Miller, B.L., Rankin, K.P., 2011. The diagnostic challenge of psychiatric symptoms in neurodegenerative disease: rates of and risk factors for prior psychiatric diagnosis in patients with early neurodegenerative disease. J Clin Psychiatry 72(2), 126-133. 